\title{
CONFINEMENT OF ENERGETIC ALPHAS IN TCT AND TRITONS IN PLT
}

\section{PLASMA PHYSICS LABORATORY}

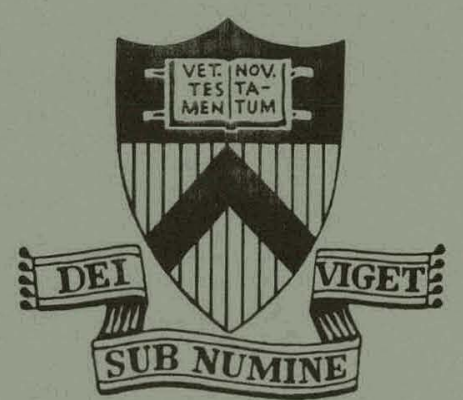

\section{PRINCETON UNIVERSITY PRINCETON, NEW JERSEY}

This work was supported by U. S. Atomic Energy Commission Contract AT (11-1)-3073. Reproduction, translation, publication, use, and disposal, in whole or in part, by or for the United States Government is permitted. 


\section{DISCLAIMER}

This report was prepared as an account of work sponsored by an agency of the United States Government. Neither the United States Government nor any agency Thereof, nor any of their employees, makes any warranty, express or implied, or assumes any legal liability or responsibility for the accuracy, completeness, or usefulness of any information, apparatus, product, or process disclosed, or represents that its use would not infringe privately owned rights. Reference herein to any specific commercial product, process, or service by trade name, trademark, manufacturer, or otherwise does not necessarily constitute or imply its endorsement, recommendation, or favoring by the United States Government or any agency thereof. The views and opinions of authors expressed herein do not necessarily state or reflect those of the United States Government or any agency thereof. 


\section{DISCLAIMER}

Portions of this document may be illegible in electronic image products. Images are produced from the best available original document. 
Confinement of Energetic Alphas

in TCT and Tritons in PLT

Frederick H. Tenney

Plasma Physics Laboratory, Princeton University

Princeton, New Jersey 08540

\section{ABSTRACT}

The nature of the drift orbits of $3.5 \mathrm{MeV}$ alpha particles in tokamak fields typical of the proposed TCT device is discussed. The drift equations are approximately integrated to obtain orbit equations. A variety of orbits: are displayed including near "stagnation" orbits.

An estimate is made of the initial confinement of 3.5 MeV alphas in TCT and $1.0 \mathrm{MeV}$ tritons in PT.

I. Introduction

In the next generation of large tokamaks such as the Princeton Large Torus (PLT), now under construction, and the proposed Two Component Torus (TCT) there is the possibility that a sizable fraction of the energetic charged products of fusion reactions occurring in these machines will not be rapidly. lost to the walls. In this report we make an estimate of this fraction for the PLT and TCT.

Both the PLT and TCT will develop discharge currents in excess of one megampere in toroidal magnetic fields of 45 
kilogauss. The minor radius of the vacuum vessel of the PLT is about $50.0 \mathrm{~cm}$ and that of the TCT about $95 \mathrm{~cm}$. These dimensions are large compared to the Larmor radius of about $6 \mathrm{~cm}$ that either a $3.5 \mathrm{MeV}$ alpha particle from the DT reaction or a $1.0 \mathrm{MeV}$ triton from the $\mathrm{DD}$ reaction would have in the toroidal magnetic field. However, because of their high energy these particles are expected to travel long distances before appreciable scattering takes place. Consequently they wili execute many arift orblts as defined by the colitsioniess motion of their gulding centers in the magnetic field of the tokamak. Some of these orbits will pass far from the discharge and into the surrounding walls. (Refs. 2 and 3) A particle born to such a drift orbit will be rapidly "lost" to the walls. Since the nature of the drift orbit will depend not only on the initial pitch angle, but also the initial position of the charged particle, a large number of initial conditions must be surveyed in order to estimate the fraction of charged particles born on "lost" orbits.. Consequently a rapid method of determining orbits is destrable.

II. Orbit Equation

We calculate only the drift orbits of energetic ions. We use cylindrical coordinates $R, z$, and $\phi$ and approximate the tokamak fields by a static magnetic field axisymmetric about the $z$ axis. The poloidal field is taken to have two constituents: one, the field arising from the discharge current that is confined to a torus of major radius $R_{0}$ and 
minor radius a; the other a uniform "vertical". field, "B্ sufficient to establish an equilibrium for: the discharge according to the formula: (Ref. 4 )

$$
B_{v}=-\frac{I}{R_{0}}\left(\ln \frac{8 R_{0}}{a}-\frac{5}{4}+B_{\theta}\right)
$$

where $I$ is the total current, and $\beta_{\theta}$ is the ratio of plasma pressure to poloidal magnetic field pressure at the plasma surface. The toroidal field, $B_{\phi}$, is taken at nominal values for the respective devices analyzed and in all cases is approximately an order of magnitude larger than the poloidal field.

Figure 1 shows the resulting poloidal field for a 1.0 MA discharge current, uniform current density, with: $R_{0}=270 \mathrm{~cm}, a=60 \mathrm{~cm}$ and $B_{\theta}=2.0$. Also shown, in dashed, lines, is the poloidal field with $B_{v}=0$. It is evident that application of the vertical field produces a marked shift in the magnetic surfaces (the magnetic surfaces coincide with the poloidal field lines in Fig. 1) causing them to be roughly concentric with the current distribution. The drift orbits are calculated according to the expression :

$$
\mathrm{qRA}_{\phi}+\mathrm{mRv}_{\|}=\text {constant }
$$

where $q$ is the charge and $m$ the mass of a particle at radius $R$ moving with guiding center speed $v_{\| l}$ parallel to the axisymmetric magnetic field. $A_{\phi}$ is the toroidal component of the vector potential of the magnetic field. The toroidal coordinate $\phi$ does not appear in Eq. 2 because of the 
assumption of axisymmetry. Thus the "orbit" defined by Eq: 2 . represents the projection of the three dimensional drift orbit onto the two dimensional $R, Z$ plane.

Equation 2 is an approximation to the exact statement that, under the assumption of axisymmetry, the toroidal component of the canonical angular momentum, $\mathbf{P}_{\phi}$, of a charged particle in a static magnetic field is a constant of the motion; that is,

$$
\mathrm{P}_{\phi} \equiv \mathrm{qRA} A_{\phi}+\mathrm{m} R \mathrm{Rv}_{\phi}=\text { constant }
$$

where $v_{\phi}$ is the $\phi$-component of the particle velocity. Equations 2 and 3 appear nearly identical. However, the variables of Eq. 3 refer to the particle itself whereas in Eq. 2 the reference is to the guiding center of the particle.

Physically, Eq. 3 states that the sum of the mechanical angular momentum of the particle about the axis of symmetry and the product of $(q / 2 \pi)$ and the poloidal magnetic fiux between the particle and the axis of symmetry is a constant. $\left(2 \pi \mathrm{RA}_{\phi}=\right.$ poloidal flux $)$ In other words, a change in mechanical angular momentum about the $Z$ axis requires a torque about the z-axis and this, in turn, requires motion of the charged particle perpendicular to the poloidal magnetic field.

Equation' 2 can be derived from Eq. 3 by averaging Eq. 3 "over a gyration period of the charged particle and making the final approximation of $v_{\|}=\left(v_{\phi}\right)$ average. This last approximation is justified when the toroidal magnetic field is much larger than the poloidal magnetic field. 
Equation 2 can also be derived by direct integration of the drift equation (Ref. 1) for the velocity of the guiding center of a charged particle. It is instructive to do so. The velocity, $\underline{\mathrm{V}}$, of the guiding center is given by $\operatorname{Ref} \cdot \dot{5}$

$$
\underline{v}=v_{\|} \frac{\underline{B}}{B}+\frac{E}{B_{B}^{2}}+\frac{m v_{\|}^{2}}{q^{4}}\left(\underline{B} \times(\underline{B} \cdot \nabla) \underline{B}+\frac{m v_{-1}^{2}}{2 q B^{3}}(\underline{B} \times \nabla B)\right.
$$

Here $v_{\|}$and $v_{\perp}$ are the speeds parallel and perpendicular to the $\underline{B}$ field of a particle of charge $q$ and mass $m$. The first term of Eq. 4 is a motion parallel to $\underline{B}$, the so-called "streaming" motion. The remaining three terms are each perpendicular to $\underline{B}$. They represent drift velocities in response to: the electric field E, the centrifugal effects of motion along a curved $\underline{B}$ line and the repulsion of a diamagnetic particle from regions of stronger magnetic field strength. The underlying supposition for Eq. 4 to be valid is that the electromagnetic field have only small variation in distances the order of the Larmor radius and in times the order of the gyration period of the charged particle. Under these assumptions the magnetic moment of the particle,

$$
\mu \equiv \frac{1}{2} \mathrm{mv}_{\perp}^{2} / \mathrm{B}
$$


is also conserved. We assume the magnetic field does not vary in time. Hence the $E$ field is electrostatic. The total energy, $U$, of the particle is conserved in the absence of collisions and is given by

$$
\mathrm{U}=\frac{1}{2} \cdot \mathrm{m}\left(\mathrm{v}_{\|}^{2}+\mathrm{v}_{\perp}^{2}\right)+\mathrm{qW}
$$

where $W$ is the electrostatic potential.

We proceed to integrate the poloidal component nf $\underline{V}$. To this end we rewrite Eq. 4 as

$$
\underline{v}=\frac{v_{l l}}{\bar{B}}\left(\hat{\phi}_{\psi}+\underline{B}_{p}\right)+\underline{V}_{D}
$$

where the three drift terms of Eq. 4 have been collected into a single drift velocity, $\underline{\nabla}_{D}$, and $\underline{B}_{p}$ is the poloidal constituent of the magnetic field. The circumflex (^) designates a unit vector. Now

$$
V_{R}=\underline{V} \cdot \hat{R}=\frac{d R}{d t}=\frac{V_{l}}{B} \cdot \underline{B}_{p} \cdot \hat{R}+\underline{V}_{D} \cdot \hat{R}
$$

and

$$
v_{z}=\underline{v} \cdot \hat{z}=\frac{d Z}{d t}=\frac{v_{l l}}{B} \cdot \underline{B} \cdot \hat{z}+\cdot v_{n} \cdot \hat{z}
$$

We express $\underline{B}_{p}$ in terms of the poloidal magnetic field flux $\psi(R, Z)$. We take $\psi(R, Z)$ to be the magnetic flux linking a circle centered on the Z-axis and passing through the point R, Z. Thus

$$
2 \pi R \underline{\mathrm{B}}_{\mathrm{p}}=\nabla \psi \times \hat{\phi}=\hat{\mathrm{R}}\left(-\frac{\partial \psi}{\partial \mathrm{z}}\right)+\hat{\mathrm{Z}}\left(\frac{\partial \psi}{\partial \mathrm{R}}\right)
$$


The time dependence is eliminated between Eqs。 7 and 8 to obtain

$$
v_{z} / v_{R}=\frac{d Z}{d R}=\frac{\underline{v}_{D} \cdot \hat{z}+\frac{v_{\| l}}{2 \pi B R} \frac{\partial \psi}{\partial R}}{\underline{v}_{D} \cdot \hat{R}-\frac{v_{\|} \cdot}{2 \pi B R} \frac{\partial \psi}{\partial z}}
$$

or

$$
\left(\mathrm{d} \underline{\underline{s}} \times \underline{v}_{D}\right) \cdot \hat{\phi}=\frac{v_{\|}}{2 \pi B R} d \psi
$$

where: $d \underline{s}=\hat{R} d R+\hat{z} d Z$ and is an element of arc length of the guiding center orbit in $R, Z$ coordinates.

We can eliminate $v_{\perp}$ from the drift velocity by the following argument. Since $\mu$ is constant we have from the gradient of Eq. 5 and using Eq. 6

$$
\frac{v_{1}^{2}}{B} \quad \nabla B=\nabla v_{\perp}^{2}=\frac{2 q}{m} \underline{E}-\nabla v_{\|}^{2}
$$

We use this expression to eliminate $v_{\perp}^{2} \nabla B$ from $\underline{v}_{D}$ to find:

$$
\underline{\mathrm{v}}_{\mathrm{D}}=-\frac{\mathrm{m}}{2 \mathrm{qB}^{2}} \underline{\mathrm{B}} \times \nabla \mathrm{v}_{\|}^{2}+\frac{\mathrm{mv}_{\|}^{2}}{\mathrm{qB}^{4}}(\underline{B} \times(\underline{B} \cdot \nabla) \underline{B})
$$

Surprisingly the explicit dependence on the $\underline{E}$ field has also been eliminated from the expression for $\underline{V}_{D}$ As shown in the 
appendix, substituting Eq. 10 into Eq. 9 results in the following equation:

$$
\begin{aligned}
& d \psi\left(1-\frac{m v_{\|} B_{\phi} B_{p}}{q B^{3} \rho}\right)=\frac{2 \pi m}{q} \frac{{ }^{B}}{B} \quad-R \cdot d v_{\|}-v_{\|} d R+\frac{B_{p}^{2}}{B^{2}} v_{\|} d R \\
& \left.+\frac{\mathrm{v}_{\|} \mathrm{R}}{\mathrm{B}^{2}} \quad \mathrm{~d} \underline{\mathrm{s}} \cdot \underline{\mathrm{B}}_{\mathrm{p}}\left(\hat{\mathrm{B}}_{\mathrm{p}} \cdot \nabla \mathrm{B}_{\mathrm{p}}+\frac{\mathrm{B}_{\mathrm{R}}}{\mathrm{R}}\right)\right\} \text {, }
\end{aligned}
$$

where $\rho$ is the radius of curvature of the poloidal field, $\underline{B}_{p}$, and $B_{R}=\underline{B}_{p} \cdot \hat{R}$

$$
\text { If } B_{p} \ll B_{\phi}, \underset{b}{E q} .11 \text { simplifies to (see appendix). }
$$

$$
\mathrm{d} \psi \stackrel{\because}{=}-\frac{2 \pi m}{\mathrm{q}} \mathrm{d}\left(\mathrm{v}_{\|} R\right)
$$

or:

Since $\psi=2 \pi R A_{\phi}, E q .12$ is equivalent to Eq. 2 .

We proceed under the assumption of no electric fields and that the toroidal magnetic field dominates. It follows that:

$$
\begin{gathered}
v_{11}^{2}+v_{\perp}^{2}=v^{2}=\text { constant }, \\
B=B(0) \frac{R(0)}{R},
\end{gathered}
$$


and

$$
\mathrm{v}_{\perp}^{2}=\frac{\dot{2} \mu}{\mathrm{m}} \mathrm{B}=\mathrm{v}_{\perp}^{2}(\mathrm{O}) \frac{\mathrm{R}(\mathrm{O})}{\mathrm{R}}
$$

where the argument. "(o)" indicates evaluation" at the "starting point" on the orbit. Thus we have

$$
\begin{aligned}
\mathrm{v}_{\|}^{2} \mathrm{R}^{2} & =\left(\mathrm{v}^{2}-\mathrm{v}_{\perp}^{2}\right) \mathrm{R}^{2} \\
& =\mathrm{v}^{2} \mathrm{R}^{2}\left(1-\frac{\mathrm{v}_{1}^{2}(0)}{\mathrm{v}^{2}} \cdot \frac{\mathrm{R}(\mathrm{o})}{\mathrm{R}}\right)
\end{aligned}
$$

Thus Eq. 12 becomes

$\Psi(\mathrm{R}, \mathrm{Z})+\alpha \cdot \frac{2 \pi m \mathrm{~V}}{\mathrm{q}} \sqrt{\mathrm{R}\left(\mathrm{R}-\mathrm{R}^{*}\right)} \equiv \mathrm{K}\left(\mathrm{R}, \mathrm{Z}, \mathrm{R}^{*}\right)=$ constant

where $\mathrm{R}^{*} \equiv \mathrm{R}(\mathrm{o}) \frac{\mathrm{v}_{1}^{2}(\mathrm{o})}{\mathrm{v}^{2}}$ and $\alpha=+1$ or -1 as $\mathrm{v}_{\|}$is in the same or opposite sense as the magnetic field. In Eq. 13 the only dependence on $\mathrm{Z}$ occurs through the initial conditions and the poloidal field flux $\psi=\psi(R, Z)$.

For tokamaks of interest with aspect ratios $\geq 3$ and "safety factors" in excess of 2 at the plasma edge we have $\mathrm{B}_{\mathrm{p}} / \mathrm{B}_{\phi} \leq 1 / 6$ throughout the volume of interest. Hence Eq. 13 is judged to be valid for drift orbit calculations in TCT and PLT in the absence of electric fields.

III. Method of Calculation

The most time consuming part of Eq. 13 to calculate is the poloidal flux in toroidal geometry. Consequently 
to evaluate Eq. 13 a $100 \times 100$ grid of points was established in the $R, Z$ coordinate system. The grid was centered on the tokamak discharge. For TCT the grid spacing was $2.4 \mathrm{~cm}$. The value of $\psi$ was determined for each point of the grid and stored for future use. The value of $\mathrm{K}$ in Eq. 13 is established for a given orbit by specifying the initial location and pitch angle of the charged particle. 'Ihe entire grid of points is then systematically scanned to find values of $R$ and $z$ that satisfy $E q .13$. Linear interpolation is used between grid points. Solutions of Eq. 13 were accepted using both the initial value of $\alpha$ and the negative of the initial value. 'In this way both "trapped" and "passing" orbits are completely traced within the grid points. In addition, "disconnected orbits" are also traced as discussed below.

Selected orbits of $3.5 \mathrm{MeV} \alpha$ particles are shown in Fig. 2 for TCT parameters. Each panel of Fig." 2 contains orbits originating from a particular point on the midplane $(z=0)$ within the TCT discharge. The small " " on the midplane at $R=270 \mathrm{~cm}$ is the center of the discharge current distribution which, in Fig, 2 , is taken as uniform and extending to a minor radius of $60 \mathrm{~cm}$. The extent of the current distribution is indicated by the circle of long dashes. The curve of short dashes labelled B in each panel is the poloidal field line passing through the origin of the orbits shown. The orbits are in full line, and labelled 
with the starting pitch angle, $\theta(0)$, where

$$
\sin ^{2} \theta(0) \equiv v_{\perp}^{2}(0) / v^{2}
$$

The sense of the toroidal magnetic field and the discharge current are taken to be into the paper and $\theta(0)=0$ corresponds to $\mathrm{v}_{\|}$: being in the same sense as: the discharge current." In the upper left panel are two dotted orbits each labelled with $110^{\circ}$ and in the upper right panel are two similar orbits labelled with $120^{\circ}$. These orbits are examples of "disconnected orbits" that represent numerical solutions of Eq. 13 .

IV. Nature of the Orbits

The orbits to be discussed are defined as the locus of points $(R, Z)$ that satisfy Eq. 13. These orbits are the projection on the $R, Z$ plane of the three dimensional drift orbits of the guiding center of a charged particle moving without collisions in a static axisymmetric magnetic field.

We make several observations about Eq. 13. First, if the poloidal flux term, $\psi$, dominates the angular momentum term the orbit will lie very close to the magnetic surface passing through the starting point of the orbit. In the limit of a massless particle the orbit coincides with the magnetic surface. 
The physical situation is: seen clearly from.Eq. 4.: The drift terms arising from non-uniform $\underline{B}$ effects depend quadratically on the particle speed. The motion along the $\underline{B}$ field line is linear in particle speed. Thus, a reduction in particle momentum, $: m v$, results in a reduction of drift motion relative to the "streaming" motion along the field lines. The particle with low speed remains closer to a given magnetic surface than if it had high speed. This effect is illustrated in Fig. 3 ; in which one can compare $1.0 \mathrm{MeV}$ orbits with $3.5 \mathrm{MeV}$, orbits that have identical initial pitch angles and starting position.

Alternatively, since the magnetic field is predominantly toroidal, an increase in poloidal field: strength results in an increase in poloidal speed of the guiding center due to its streaming motion without affecting the drift speed. Hence an increase in poloidal field strength results in a drift orbit remaining closer to the magnetic surface on which it started. This effect is illustrated in Fig.: 4 in which one can compare $3.5 \mathrm{MeV}$ alpha orbits in a uniform and a non-uniform current distribution. The non-uniform current distribution is of the form

$$
J(r)=J(0)\left(1-r^{2} / a^{2}\right)^{3 / 2}
$$


where $r$ is the minor radius of the discharge. Since the total current is the same for both current distributions the polodial fields of these two distributions will differ most near the center of the discharge, the non-uniform current distribution producing the larger polodial field. Consequently the orbits in the right hand panels of Fig. 4 (which originate at about $r / a=0.66$ ) are not as affected as are the orbits in the left hand panels (originating at about $\mathrm{r} / \mathrm{a}=0.20)$.

Second; R must not be less than $R^{*}$ in. order for the square root in Eq. 13 to remain a real quantity. $R=R^{*}$ implies $v_{\|}=0$. At such a point the orbit in the $R, Z$ plane will be parallel to the Z-axis and the particle will be undergoing reflection in its motion parallel to the magnetic field. Particles which undergo such reflection are called "trapped" particles in tokamak parlance. All other particles are called "passing" particles. For example, in Fig. 2 the orbits for $\theta(0)=100^{\circ}$ are trapped particle orbits and those for $\theta(0)=0^{\circ}$ are passing particle orbits. The trapped particle orbits have characteristic "banana" shapes.

Third, two orbits starting with the same values of $R(0), Z(0)$, and $R *$ are distinguished from each other only by the sense of $v_{\|}(0)$ which determines the initial sign of $\alpha$. Reversing the sense of the discharge current and hence the 
sign of $\psi$, and also reversing the sense of $v_{\|}(0)$, and hence the sign of $\alpha$, results in no change in the orbit defined by Eq. 13. Thus the orbits are independent of the sense of the toroidal field but do depend on the sense of $v_{\|}$(o) relative to the sense of the discharge current. particles which are "injected" with $\vec{v}_{\|}$(o) in the same (opposite) sense as the discharge current are said to be co(counter) - injected.

Fourth, ror the tokamak configuration $\psi(K, Z)$ is symmetric in $z$. Therefore all orbits w111 be symmetric in Z.

Each of the orbits displayed in Figs. 2-4 can be rationalized in terms of the drift motion and streaming motion of the charged particle. In the approximation of strong toroidal field the drift motion will always be purely "vertical", that is, in the +z direction. The streaming motion along a tield line results in a motion along a poloidal "field line in either a clockwise or counterclockwise sense about the magnetic axis depending on the sense of $v_{\|}$' For example, consider the orbits in Fig. 2 in the upper left panel. The $50^{\circ}$ orbit starts out in the $+Z$ direction since both the drift motion and the streaming motion are in that direction. The streaming motion tends to make the orbit follow the polodial B line passing through the starting point. The drift motion causes the orbit to rise above the $B$ line. The streaming motion dominates and 
hence the orbit eventually bends over and back to the $\mathrm{Z}=0$ plane. Continuing into negative $Z$ the drift motion now pulls the orbit closer to the starting B line in such a manner as to produce an orbit symmetric in $Z$. The orbit is executed in a clockwise sense.

Consider the $100^{\circ}$ orbit in the same panel. Since $\theta(0)>90^{\circ}$ the streaming motion will be in a counter clockwise sense and hence initially in a $-\mathrm{Z}$. direction. However, for $\theta(0)=100^{\circ} \quad v_{\|}$will be small enough so that the drift motion will dominate and hence the orbit will start out in the $+Z$ direction. For positive $Z$ the streaming motion will have a component in the $-\mathrm{R}$ direction. This motion added to the vertical drift motion results in the orbit bending over to smaller values of $R$ and hence into a region of stronger toroidal field. The value of. $v_{\|}$is thereby reduced, and the particle eventually "mirrors" in the toroidal field. At the "mirror" point $\eta_{l}$ vanishes and the motion along the orbit is purely vertical drift motion. The vertical motion across the poloidal field produces a force on the particle in the positive toroidal sense which causes $\mathrm{v}_{\|}$to become positive. The streaming motion now produces a clockwise motion which, in conjunction with the drift motion, produces a segment of orbit similar in character to the $50^{\circ}$ orbit discussed above. The particle is eventually carried into a second mirror point symmetric in $Z$ with the first mirror point already discussed. After mirroring the 
particle moves in a negative toroidal sense that produces,... since $Z$ 'is negative, a streaming motion with a component of: motion in the $+R$ direction: This motion added to the : dominant vertical drift motion completes the closed orbit. Consider the $130^{\circ}$ orbit in the same panel. Here $v_{\|}$ is sufficiently negative so that the streaming motion dominates the drift motion, and the orbit, starts out in the $-Z$ direction. The streaming motion will tend to make the orbit follow the poloidal field line passing through the starting point in a counterclockwise sense.: The vertical drift:motion causes the orbit to rise above the $B$. line. Thus the particle enters a region of weaker toroidal field and weaker poloidal field. The reduction in poloidal field strength is apparently sufficient to reduce the $R, Z$ motion due to the streaming motion to such an extent that the drift, motion dominates, and the orbit bends up to the midplane without encircling the magnetic axis. The orbit at the midplane is directed in the, $+\mathrm{Z}$ direction while $\mathrm{v}_{\|}$is still negative: For positive values of $\mathrm{Z}$ the streaming motion will have a $-R$ component which results in the orbit bending to the left. The orbit, therefore, moves into a region of stronger poloidal field. The counter clockwise streaming motion is enhanced and eventually dominates the drift motion to close the orbit on its starting point.

It is clear that for some value of $100^{\circ}<\theta(0)<130^{\circ}$ the downward.streaming motion and the upward drift motion will 
just cancel. The guiding center of the particle will then simply describe a circular orbit in the midplane centered on the $\mathrm{Z}$ axis. Such a "point orbit" will be called a stagnation orbit and its starting pitch angle will be called: the stagnation pitch angle, $\theta_{S}(0)$. Other examples in Fig. 2 of a stagnation orbit are: the starting point in the lower left panel for which $\theta_{\mathrm{S}}(0)$ lies between $0^{\circ}$ and $50^{\circ}$; and the starting point in the lower right panel for which $\theta_{S}$ (o) lies between $50^{\circ}$ and $80^{\circ}$. The starting point in the upper right panel cannot be a stagnation orbit since the drift motion is larger than the streaming motion for all pitch angles.

Passing through these stagnation orbits is a characteristic. "pattern of elliptically " shaped orbits generated by values of $\theta(0)$ near the value of the stagnation pitch angle. The most striking illustration of such a family of orbits in Fig. 2 are the $0^{\circ}$ and $50^{\circ}$ orbits in the lower left panel. In the upper left panel the $130^{\circ}$ orbit and the small loop of the $110^{\circ}$ orbit are also part of a family of eliptic orbits. However the $110^{\circ}$ disconnected orbits, and the $120^{\circ}$ disconnected orbits shown in the upper right panel of Fig. 2, suggest the existence of a second type of stagnation orbit. Such a stagnation orbit would lie on the midplane about halfway between the two disconnected orbits. Orbits in the neighborhood of such stagnation orbits are shown in Fig. 5. Their hyperbolic character is apparent. 
An analytic description of stagnation and near-stagnation orbits is easily obtalned from Eq. $130^{\circ}$ Expanding $K\left(R, Z, R^{*}\right)$ about the starting point $[(R(0), Z(0))$ we have to second order in the displacement:

$$
\frac{\partial K}{\partial R} r+\frac{\partial K}{\partial Z} z+\frac{1}{2} \frac{\partial^{2} K}{\partial R^{2}} r^{2}+\frac{\partial^{2} K}{\partial R \partial Z} r z+\frac{1}{2} \frac{\partial^{2} K}{\partial z^{2}} z^{2}=0
$$

where

$$
\begin{aligned}
& r \equiv R-R(0) \\
& z \equiv Z-Z(0)
\end{aligned}
$$

and the partial derivatives of $K\left(R, Z, R^{*}\right)$ are evaluated at $(R(0), Z(O))$. If we further restrict ourselves to starting points near the $\mathrm{Z}=0$ plane,'we can expand the partial derivatives about the point $(R(0), 0)$. By virtue of the assumed symmetry of $K\left(R, Z, R^{*}\right)$ in $Z$ all partial derivatives of $K$ involving odd orders in $\mathrm{Z}$ must vanish when evaluated at $\mathrm{Z}=0$. Thus Eq. 14 becomes:

$$
\mathrm{Ar}^{2}+\mathrm{Brz}+\mathrm{Cz}^{2}+\mathrm{Dr}+\mathrm{Ez}=0
$$


where

$$
\begin{aligned}
& A=\frac{1}{2} \partial^{2} \mathrm{~K} / \partial \mathrm{R}^{2} \\
& \mathrm{~B}=\left(\partial^{3} \mathrm{~K} / \partial \mathrm{R} \partial \mathrm{Z}^{2}\right) \mathrm{Z}(\mathrm{O}), \\
& \mathrm{C}=\frac{1}{2} \partial^{2} \mathrm{~K} / \partial \mathrm{Z}^{2}, \\
& \mathrm{D}=\partial \mathrm{K} / \partial \mathrm{R}, \\
& \mathrm{E}=\left(\partial^{2} \mathrm{~K} / \partial \mathrm{Z}^{2}\right) \mathrm{Z}(\mathrm{O})=2 \mathrm{C} \mathrm{Z}(\mathrm{O})
\end{aligned}
$$

and where the partial derivatives are evaluated at the point $(R(0), 0)$

Equation 15 describes orbits that are conic sections. In particular for $\mathrm{Z}(\mathrm{O})=0 \mathrm{Eq} \cdot 15$ reduces to:

$$
\mathrm{Ar}^{2}+\mathrm{Cz}^{2}+\mathrm{Dr}=0
$$

If: the : coefficients $A$ and $C$ are of the same sign, Eq. 16 describes a family of ellipses as shown by the solid line curves in Fig. 6a. If $A$ and $C$ are of opposite sign, Eq. 16 describes a family of hyperbolae as shown by the solid line curves in Fig. 6b. For $D \rightarrow 0$, the ellipses shrink to a point and the hyperbolae approach the asymptotes. In either case for $D \neq 0$ the orbits cross the $Z=0$ plane in a perpendicular direction.

An expression for the slope of the orbits follows from differentiating Eq. 15 with.respect to $r$. We find

$$
d z / d r=-\frac{[2 A r+B z+D]}{[B r+2 C z+E]}
$$


At the starting point $(0,0)$ we have:

$$
(\mathrm{dz} / \mathrm{dr})_{\mathrm{O}, \mathrm{o}}=-\mathrm{D} / \mathrm{E}=-\frac{\partial \mathrm{K} / \partial \mathrm{R}}{\left(\partial^{2} \mathrm{~K} / \partial \mathrm{Z}^{2}\right) \mathrm{Z}(\mathrm{O})}
$$

From Eq. 18 it is clear that for orbits that cross the $Z=0$ plane (hence we can chose $\mathrm{Z}(-0)=0$ ) the crossing slope is always infinite unless $D=0$. For orbits with $Z(0) \neq 0$, but with $R(0)$ such that $D$ vanishes we have $(d z / d r)_{0,0}=0$ and the orbit starts out parallel to the $\mathrm{Z}=0$ plane. Such orbits are described by setting $D=0$ in Eq. 15. Neglecting the second term containing $\mathrm{B}, \mathrm{Eq} .15$ then becomes

$$
\mathrm{Ar}^{2}+\mathrm{Cz}^{2}+\mathrm{Ez}=0
$$

If $A / C<0$ Eq. 19 describes the family of hyperbolae shown by dotted lines in Fig. $6 \mathrm{~b}$. If $\mathrm{A} / \mathrm{C}>0 \mathrm{Eq} .19$ describes a family of ellipses one of which is shown. by a dotted line in Fig. 6a.

The two conditions that characterize the limiting orbits in Fig. 6 are: .

$$
\mathrm{D} \equiv \partial \mathrm{K} / \partial \mathrm{R}=0
$$

and the sign of A/C. The physical meaning of $D=0$ is the vanishing of the $\mathrm{Z}$ component of orbital velocity on the midplane that will occur if the upward drift motion is just 
balanced by a downward streaming motion. To see this analytically we set the partial derivative of Eq. 13 equal to zero, namely:

$$
\partial K / \partial R=\partial \psi / \partial R+\frac{2 \pi m}{q} \frac{\partial}{\partial R}\left(v_{\|} R\right)=0
$$

To evaluate the second term of Eq. 20 we turn to the drift velocity, $\underline{V}_{D}$, and evaluate Eq. 10 under our assumption that the magnetic field is approximated by just the toroidal field, $\hat{\phi}_{\phi}$ : Under this assumption, we recall, $v_{\|}$is not a function of $Z$. We find

$$
\underline{v}_{D}=\hat{z} \frac{m v_{\|}}{q B_{\phi}} \quad \frac{1}{R} \quad \frac{\partial}{\partial R}\left(v_{\|} R\right)
$$

This result, together with the relation

$$
\dot{\partial} \psi / \partial R=2 \pi R B_{Z}
$$

substituted into $\mathrm{Eq}$. 20 yields the relation

$$
v_{D}=-v_{\| l} \frac{B_{z}}{B_{\phi}}
$$

We recognize the right hand side of this relation to be just the negative of the $\mathrm{z}$-component of the streaming motion in the $R, Z$ plane. 'lihus when $\partial \mathrm{K} / \partial \mathrm{R}$ vanishes the net $\mathrm{Z}$-component of the orbital motion in the $R, Z$ plane : vanishes. However, when $\partial \mathrm{K} / \partial \mathrm{R}$ vanishes on the midplane, that is when $\mathrm{D}=0$, 
both the $R$ and $Z$ components of orbital motion will vanish. One concludes that there is no motion in the $R, Z$ plane along. the stagnation orbits.

The physical meaning of the sign of A/C relates to the curvature of $K\left(R, Z, R^{*}\right)$ for $Z=0$. Now

$$
\mathrm{C} \equiv \cdot \frac{1}{2} \partial^{2} \mathrm{~K} / \partial \mathrm{Z}^{2}=\frac{1}{2} \partial^{2} \psi / \partial \mathrm{Z}^{2}
$$

evaluated for $z=0$. From inepeotion of Fig: 1 il is clear. that the value of $\psi$ will be reduced as one moves of the $Z=0$ plane in either direction. Hence $C<0$ for any $R$. Thus the nature of the stagnation orbits will vary as the sign of A varies.

To illustrate the nature of $A$ we have plotted in Fig. 7 the function $K\left(R, 0, R^{*}\right)$, defined by $E q$. 13, evaluated for a $3.5 \mathrm{MeV}$ alpha particle and the same magnetic field parameters that apply for Fig. 2. The parameter for the family of curves is $R^{*}$. The curve of poloidal field flux $\psi(R, 0)$, that is the first term of $K\left(R, 0, R^{*}\right)$, serves to divide the curves of $K\left(R, 0, R^{*}\right)$ into an upper branch that lies above $\psi(R, 0)$ and a lower branch that lies below. The upper branch is produced by setting $\alpha=+1$, the lower branch by $\alpha=-1$. As stated earlier we have chosen both the discharge current and the toroidal magnetic field to be in the $+\hat{\phi} \cdots$ sense, and therefore $\alpha=+1$ corresponds to "co-injected" particles. Furthermore, $\psi(R, 0)$ will be, 
therefore, a positive quantity. It increases from zero at the $\mathrm{Z}$ axis of symmetry to a positive maximum at the magnetic axis and falls to zero once more at the separatrix.. This behavior is obviously consistent with the poloidal field pattern shown in Fig. 1 if the poloidal field is taken to act in a clockwise sense.

Each $K\left(R, 0, R^{*}\right)$ curve corresponds to a particular. choice of $R^{*}$ which will be the value of $R$ at which the $\psi(R, 0)$ curve is crossed. It is clear from inspection. of Fig. 7 where $D$, vanishes. Where the curve of $K\left(R, 0, R^{*}\right)$ is concave downward (upward) A will be negative (positive). For example, at points $a, b$ and $c$ in Fig. 7 we have the conditions satisfied for stagnation orbits. At points a and $b$ the stagnation orbit is of the elliptic type whereas at point $c$ the orbit is of the hyperbolic type. The dotted curves are the loci of stagnation orbits. We note that for starting points near the magnetic axis it is not possible to have a stagnation orbit. The physical reason is that near the magnetic axis the poloidal field is so weak that the drift motion will dominate the streaming motion at any pitch angle.

In addition to identifying stagnation orbits a general sense of an orbit can be inferred from the plot of $\mathrm{K}\left(\mathrm{R}, 0, \mathrm{R}^{*}\right)$. For example, consider the orbit with initial parameters given. by point d in Fig. 7. This particular orbit starts on the $\mathrm{Z}=0$ plane $\underset{*}{\text { at }} \mathrm{R}(\mathrm{O}) \approx 281 \mathrm{~cm}$ and has an 
initial pitch angle such that $R^{*} \approx 264 \mathrm{~cm}$. Since point d lies above the $\psi(R, 0)$ curve, $\alpha$ will be +1 , and $\theta(0)$ is calculated as $76^{\circ}$ rather than $104^{\circ}$. Now this orbit in the $R, Z$ 'space 'will be' defined by the equation $K\left(R, Z, R^{*}\right)=$ $\mathrm{K}(281,0,264) \equiv \mathrm{K}(\mathrm{d})$. The values of $\mathrm{R}$ where the orbit: crosses the $Z=0$ plane are given by where the curve $K(R, 0,264)$ in Fig. 7 takes on the value of $K(d):$ This occurs at points $d$ and $d^{\prime}$. The range of $R$ for which this orbit is defined will be characterized by the condition $K(R ; 0,264) \geq K(d)$. The validity of this condition will be recognized by observing that for the magnetic fields under consideration we have $\psi(R, Z) \leq \psi(R, 0)$. It follows that $\mathrm{K}\left(\mathrm{R}, \mathrm{Z}, \mathrm{R}^{*}\right)<\mathrm{K}\left(\mathrm{R}, 0, \mathrm{R}^{*}\right)$ and hence the orbit requirement $K(R, Z, 264)=K_{d}$ can be satisfied for $Z \neq 0$ only for those values of ' $R$ for which $K(R, 0,264) \geq K(d)$. The "expected orbit through point $d$ is thus similar to the $80^{\circ}$ orbit in the lower left panel of Fig. 2.

For the orbit starting at point e in Fig. 7 we have the same values for $R(0)$ and $R *$ as for the orbit through point d above. However, since point e lies below the $\psi(R, 0)$ curve, the initial pitch angle will be $104^{\circ}$. The range of $R$ for which the orbit is defined extends from $\mathrm{R}^{*}$, at $264 \mathrm{~cm}$, to : approximately $360 \mathrm{~cm}$ at point $e^{\prime}$. This is a "trapped particle" orbit and is similar to the $100^{\circ}$ orbit in the lower left panel of Fig. 2. The particle effectively 
"oscillates" between points $e$ and $e^{\prime}$ via the reflection point at $\mathrm{R}^{*}$.

Finally consider an orbit starting at point $f$ in Fig. 7. Here $R(0) \approx 258 \mathrm{~cm}, \mathrm{R}^{*}=192 \mathrm{~cm}$ and $\theta(0) \approx 120^{\circ}$. Clearly an orbit for a "passing particle" can be defined between points $f$ and $f^{\prime}$ for which $v_{\|}<0$ everwhere. Between points $f^{\prime}$ and $f^{\prime \prime}$ we have the relation $K(R, 0,192)<K(258,0,192) \equiv K(f)$. Hence no orbit can be defined for which $v_{\|}<0$. However at point $f^{\prime \prime}$ a "trapped particle" orbit can be defined with the same value of "orbit constant" $K(f)$. This trapped particle orbit is "disconnected" from the "passing" orbit by the radial distance between points $f^{\prime}$ and $f^{\prime \prime}$. These orbits are essentially the $120^{\circ}$ orbits shown as dotted curves in the upper right panel of Fig. 2 .

From the general pattern of the curves in Fig. 7 it appears that "co-injected" particles cannot have "trapped" orbits and that their excursions in $R$ tend to "oscillate" about a position at somewhat larger radius than the magnetic axis. This outward shift in the mean radial position of "co-injected" particles results in their experiencing an average vertical magnetic field in the $-\mathrm{Z}$ direction: Such a field is necessary to provide the centripetal force required to contain the alpha particles circulating in a counter clockwise sense about the $Z$ axis, By comparison the "counter-injected". particles can have all types of orbits 
many of which lead to large radial excursions. Thus it is." more probable that a "counter-injected" alpha particle will be "lost" to the walls via a large drift orbit than for a "co-injected" alpha particle to do so. It is also evident. from the: general pattern. of : curves in. Fig. 7 that the "counter-injected" alphas that are not "trapped". have orbits: with radial excursions that tend to "oscillate" about: a position at somewhat smaller radius than the magnetic axis. This inward shift in the mean radial position of these particles results in their experiencing an average vertical magnetic field in the $+Z$ direction. Such a field is necessary to provide the centripetal force required to contain the alpha particles circulating in a clockwise sense about the $\mathrm{Z}$ axis.

V. Orbit Statistics

To determine the fraction of $3.5 \mathrm{MeV}$ alpha particles. that would be : promptly lost from the TCT device a survey of several tens of thousands of drift orbits was made. The results are shown in Fig. 8. The alphas were assumed to be born isotropically in velocity space and to have a birth distribution. in configuration space that was a function oniy of the minor radius centered at $R=270 \mathrm{~cm}$. The form of the alpha source function is given by the dashed curve labelled $S(\alpha)$ in Fig. 8 and models the effect: of the injected beam of 
deuterons being peaked on the magnetic axis. Three different discharge current distributions were assumed for a fixed total current of $1.0 \mathrm{MA}$. The non-filamentary current distributions were limited in radial extension to $60 \mathrm{~cm}$ which was the nominal minor diameter of the plasma. The toroidal magnetic field is $45 \mathrm{kG}$ at the center of the plasma.

The birth points for the alpha particles was a sub-set of the grid of points used for calculating $\psi(R, Z)$ that was described in Section III. The result for each point was weighted according to the source function $S(\alpha)$. At each birth point orbits were calculated for a variety of birth pitch angles, $\theta(0)$. The results for each pitch angle were weighted by a sin $\theta(0)$ distribution function in accordance with the assumption of isotropy of birth in velocity space. (The orientation of the initial velocity vector about the magnetic field line for a given pitch angle. is averaged out in the drift equations and does not appear in our formulation.)

For each orbit the maximum distance of the calculated orbit points from the center of the plasma was determined and provided the basis for statistical analysis. The information presented in Fig. 9 is independent of the source function $S(\alpha)$. Folding $S(\alpha)$ into the data in Fig. 9 produces the curves in Fig. 8. 
The above analysis was also applied to the orbits of 1.0 MeV tritons that are expected in the PLT device. . The results are shown in Fig. 10. The plasma minor radius was taken as $45 \mathrm{~cm}$ and an uniform current distribution assumed. The toroidal magnetic field was assumed to be $45 \mathrm{kG}$ at the center of the plasma.

\section{Acknowledgement}

The author acknowledges the very. helpful computer programming of K. K. Tang. This work was supported by Energy Research and Development Administration (Formerly Atomic, Energy Commission) Contract AT(11-1)-3073; also use was made of computer facilities supported in part by National Science Foundation Grant GP 579. 


\section{References}

1. A. I. Morozov and L. S. Solov'ev, Integrals of the Drift Equations, Dokl. Akad. Nauk SSSR 128, 506 (1959). . (Translation: Soviet Phýs. - Dokilady 4 , $1031,1960$. )

2. T. H. Stix, Heating of Toroidal Plasmas by Neutral Injection, Plasma Physics 14, 367 (1972).

3. D. G. McAlees and H. K. Forsen, Thermonuclear Alpha Particle Orbits in a Tokamak, Bull. Am. Phys. Soc 18, 1305 (1973).

4. J. M. Greene, J. L. Johnson, and K. W. Weimer, Tokamak Equilibrium, Phys. Fluids 14, 671 (1971).

5. C. L. Longmire, Elementary Plasma Physics (Interscience Publishers, New York, 1963) p. 50 . 


\section{Appendix}

We indicate the passage to Eq. 11 in the text from the irelation:

$$
\therefore \quad\left(\mathrm{d} \underline{\mathbf{s}} \times \underline{\mathrm{v}}_{\mathrm{D}}\right) \cdot \hat{\phi}=\frac{v_{\|}}{2 \pi B R} d \psi
$$

where $\underline{d} \underline{s}=\hat{R} d R+\hat{Z} d Z$ is an element of an orbit and

$$
\begin{gathered}
\mathrm{V}_{\mathrm{D}}=-\frac{\mathrm{m}}{2 \mathrm{qB}} \cdot \hat{\mathrm{B}} \times \nabla \mathrm{v}_{\|}{ }^{2}+\frac{\mathrm{mv}_{\|}{ }^{2}}{\mathrm{qB}^{4}}(\underline{\mathrm{B}} \times(\mathrm{B} \cdot \nabla) \underline{\mathrm{B}}) \\
\quad \therefore \quad(\mathrm{A} 2)
\end{gathered}
$$

We write:

$$
\begin{aligned}
& \mathrm{d} \psi=\frac{2 \pi \mathrm{BR}}{\mathrm{v}_{\|}} \mathrm{d} \underline{\mathrm{s}} \cdot\left(\underline{\mathrm{v}}_{\mathrm{D}} \times \hat{\phi}\right) \\
& =-\frac{\pi R m}{v_{d} q \mathrm{qB}} \mathrm{d} \underline{\mathrm{s}} \cdot\left(\left(\underline{\mathrm{B}} \times \nabla_{\because}{ }^{2}\right) \times \hat{\phi}\right) \\
& +\frac{2 \pi R m v_{\| 1}}{\mathrm{qB}^{3}} \mathrm{~d} \underline{s} \cdot((\underline{B} \times(\underline{B} \cdot \nabla) \underline{B}) \times \hat{\phi}) \\
& =\frac{\pi R m}{q \bar{B}}\left\{+\frac{1}{v_{\|}} d \underline{s} \cdot\left(\hat{\phi} \times\left(\underline{B} \times \nabla v_{\|}^{2}\right)\right)\right. \\
& \left.-\frac{2 v_{\|}}{B^{2}} d \underline{s} \cdot(\hat{\phi} \times(\underline{B} \times(\underline{B}: \nabla) \underline{B}))\right\}
\end{aligned}
$$


Expanding the first term of Eq. A3 we have:

$$
\hat{\phi} \times\left(\underline{B} \times \nabla v_{\|}^{2}\right)=\hat{\phi} \cdot \nabla v_{\|}^{2} \underline{B}-\hat{\phi} \cdot \underline{B} \nabla v_{\|}^{2} .
$$

But $\hat{\phi} \cdot \nabla \mathrm{v}_{\|}^{2}=\frac{1}{\mathrm{R}} \frac{\partial}{\partial \phi} \quad \mathrm{v}_{\|}^{2}=0$.

$$
\therefore \mathrm{d} \underline{\underline{s}} \cdot\left(\hat{\phi} \times\left(B \times \nabla \mathrm{v}_{\|}^{2}\right)\right)=-\mathrm{B}_{\phi} \mathrm{d} \underline{\mathrm{s}} \cdot \nabla \mathrm{v}_{\|}^{2}=-\mathrm{B}_{\phi} \mathrm{d}\left(\mathrm{v}_{\|}^{2}\right) .
$$

Expanding the second term we have:

$$
\hat{\phi} \times(\underline{B} \times \underline{B} \cdot \nabla \underline{B})=(\hat{\phi} \cdot(\underline{B} \cdot \nabla \underline{B})) \underline{B}-(\hat{\phi} \cdot \underline{B}) \underline{B} \cdot \nabla \underline{B} \cdot
$$

Taking $\underline{B}=\hat{\phi} B_{\phi}+\underline{B}_{p}=\hat{\psi} B_{\phi}+\hat{R} B_{R}+\hat{z} B_{Z}$

we have: $\underline{B} \cdot \nabla \underline{B}=-\hat{R} \frac{B_{\phi}^{2}}{R}-\hat{\phi} \frac{B_{\phi} B_{R}}{R}+\underline{B}_{p} \cdot \nabla \underline{B}_{p}$.

We write: $\quad \underline{B}_{p} \cdot \nabla \underline{B}_{p}=\underline{B}_{p} \cdot \nabla \hat{B}_{p} B_{p}=B_{p}^{2} \hat{B}_{p} \cdot \nabla \hat{B}_{p}+\underline{B}_{p} \hat{B}_{p} \cdot \nabla B_{p}$. Furthermore: $\quad B_{p}^{2} \hat{B}_{p} \cdot \nabla \hat{B}_{p}=\left(\hat{\phi} \times \frac{\left.\hat{B}_{p}\right) B_{p}^{2}}{\rho}\right.$

$$
\text { where } \rho=\text { radius of curvature of } \underline{B}_{p} \text {. }
$$

Thus: $\hat{\phi} \times(\underline{B} \dot{x}-\nabla \underline{B})=-\frac{\mathrm{B}_{\phi} \mathrm{B}_{\mathrm{R}}}{\therefore \mathrm{R}}\left(\hat{\phi} \not \hat{\phi}_{\phi}+\underline{\mathrm{B}}_{\mathrm{p}}\right)-\mathrm{B}_{\phi}\left[-\hat{\mathrm{R}} \frac{\mathrm{B}_{\phi}^{2}}{\mathrm{R}}-\hat{\phi} \frac{\mathrm{B}_{\phi} \mathrm{B}_{\mathrm{R}}}{7 \mathrm{R}}\right.$

$$
\begin{gathered}
\left.+\left(\hat{\phi} \times \hat{B}_{p}\right) \frac{B_{p}^{2}}{\rho}+\underline{B}_{p} \hat{B}_{p} \cdot \nabla B_{p}\right] \\
=+\hat{R} \frac{B_{\phi}^{3}}{R}+\left(\hat{\phi} \times \frac{\hat{B}_{p}}{\rho}\right) B_{p}^{2} B_{\phi}-B_{\phi} \underline{B}_{p}\left(\hat{B}_{p} \cdot \nabla B_{p}+\frac{B_{R}}{R}\right) .
\end{gathered}
$$


Hence: ds $\cdot \hat{\phi} \times(\underline{B} \times \underline{B} \cdot \nabla \underline{B})=\frac{B_{\phi}^{3} d R}{R_{w}}-\frac{B_{\phi} B}{2 \pi R p} d \psi-B_{\phi} d \underline{s} \cdot \underline{B}_{p}\left(\hat{B}_{p} \cdot \nabla B p\right.$ $\left.+\frac{B_{R}}{R}\right)$.

Eq.A3 reads:

$\mathrm{d} \psi=\frac{\pi \mathrm{RmB}_{\phi}}{\mathrm{qB}} \quad\left\{-2 \mathrm{dv}_{\|}-2 \mathrm{v}_{\|} \frac{\mathrm{B}_{\phi}^{2}}{\mathrm{~B}^{2}} \quad \frac{\mathrm{dR}}{\mathrm{R}}+\frac{\mathrm{v}_{\|} \mathrm{B}_{\mathrm{p}} \mathrm{d} \psi}{\pi R \mathrm{~B}^{2} \dot{\mathrm{p}}}\right.$

$\left.+\frac{2 v_{\| l}}{B^{2}} d \underline{s} \cdot \underline{B}_{p}\left(\hat{B}_{p} \cdot \nabla B_{p}+\frac{B_{R}}{R}\right)\right\}$

or:

$\mathrm{d} \psi\left(1-\frac{\mathrm{mv}_{\| 1} \mathrm{~B}_{\phi} \mathrm{B}_{\mathrm{p}}}{\mathrm{qB}^{3}{ }_{\rho}}=-\frac{2 \pi m \mathrm{~m}_{\phi}}{\mathrm{qB}}\left\{\mathrm{Rdv} v_{\|}+\frac{\mathrm{B}_{\phi}^{2}}{\mathrm{~B}^{2}} v_{\| t} \mathrm{dR}\right.\right.$

$\left.-: \frac{\dot{v}_{\|} R}{B^{2}} d \underline{d}: \underline{B}_{p}\left(\dot{\hat{B}}_{p} \cdot \nabla B_{p}+\frac{B_{R}}{R}\right)\right\} 。$

Noting that:

$R d v_{\|}+\frac{B_{\phi}^{2}}{B^{2}} v_{\|} d R=R d v_{\|}+v_{\|} d R-\frac{B_{p}^{2}}{B^{2}} v_{\|} d R$,

we recognize that Eq. A4 is identical with Eq. 11 in the text. 
For $\mathrm{B}_{\mathrm{p}}<\mathrm{B}_{\phi}$ considerable simplification of $\mathrm{Eq}$. A4 is possible. Consider the term:

$$
\frac{m v_{\|} B_{\phi} B_{p}}{q B^{3} \rho}=\frac{r_{\|}}{\rho} \cdot \frac{B_{p}}{B_{\phi}\left(1+B_{p}^{2} / B_{\phi}^{2 j}\right)} \equiv T
$$

where $r_{\|} \equiv\left(m v_{\|}\right) / q B$.

Clearly for $r_{1} / \rho=O(1)$ the condition $B_{p} / B_{\phi} \ll 1$ implies $T \ll 1$. Near the magnetic axis $\rho$ may approach zero. However, within the tokamak discharge we have

$$
\frac{\mathrm{B}_{\mathrm{p}}}{\mathrm{B}_{\phi}} \approx \frac{\mathrm{r}}{\mathrm{R}} \cdot \frac{1}{\bar{q}}
$$

where $\bar{q}$ is the "safety factor." Therefore near the magnetic axis we have

$$
\mathrm{T} \approx \frac{\mathrm{r}_{\|}}{\rho} \cdot \frac{\rho}{\mathrm{R} \overline{\mathrm{q}}} \frac{1}{\left(1+\mathrm{B}_{\mathrm{p}}^{2} / \mathrm{B}_{\phi}^{2}\right)} \approx \frac{\mathbf{r}_{\|}}{\mathrm{R} \overline{\mathrm{q}}}
$$

For tokamaks $\overline{\mathrm{q}}>1$ and $\mathrm{r}_{\|} \ll \mathrm{R}$ and hence $\mathrm{T} \ll 1$.

To show that the last two terms of Eq. A4 can be neglected compared to $d \psi$ we make the following approximation

$$
\hat{\mathrm{B}}_{\mathrm{p}} \cdot \nabla \quad \mathrm{B}_{\mathrm{p}} \leq \frac{\mathrm{B}_{\mathrm{p}}}{! \rho}
$$


and recognize that $2 \pi R \cdot\left|\mathrm{ds}_{-} \cdot \mathrm{B}_{\mathrm{p}}\right| \leq|\mathrm{d} \psi|$. Then the last two terms of Eq. A4 can be approximated as less than the following expression.

$$
\frac{r_{\| l}}{\rho} \frac{\mathrm{B}_{\mathrm{p}}^{\prime}}{\mathrm{B}_{\phi}\left(1+\mathrm{B}_{\mathrm{p}}^{2} / \mathrm{B}_{\phi}^{2}\right)} \mathrm{d} \psi \approx \mathrm{T} \mathrm{d} \psi \ll \mathrm{d} \psi \ldots
$$

Thus for $B_{p} \ll B_{\phi}$ Eq. A4 reduces to:

$$
\mathrm{d} \psi=-\frac{2 \pi \mathrm{m}}{\mathrm{q}} \mathrm{d}\left(\mathrm{Rv_{ \| }}\right)
$$

$\because \cdots x^{3}$

Equation $\mathrm{A} 6$ is identical with $\mathrm{Eq} .12$ in the text. 


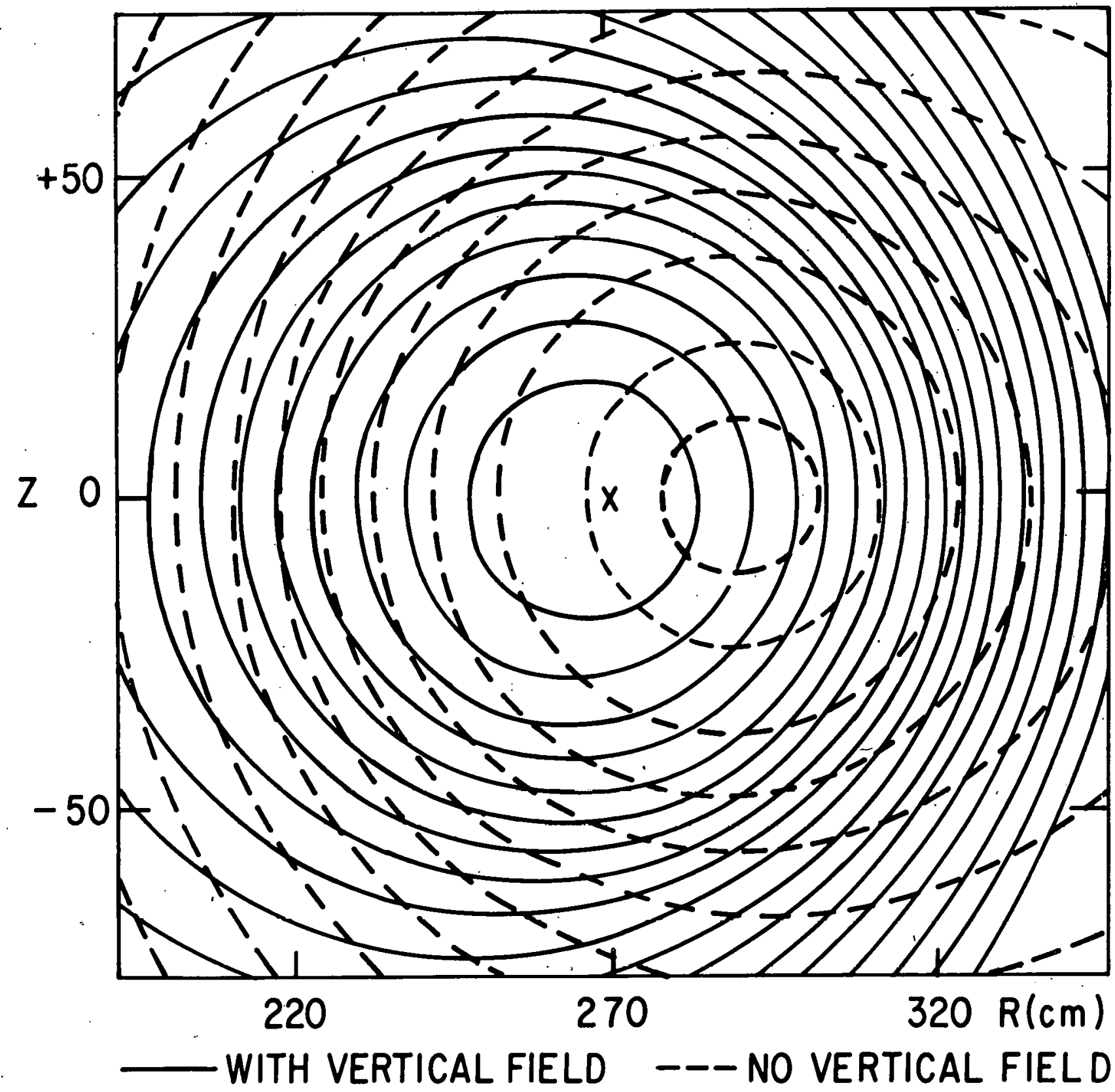

MAGNETIC SURFACES OF TOROIDAL CURRENT,UNIFORM J.

$744593^{\circ}$

.Fig. 1. Magnetic Surfaces of Toroidal Current With Uniform Current Distribution. 

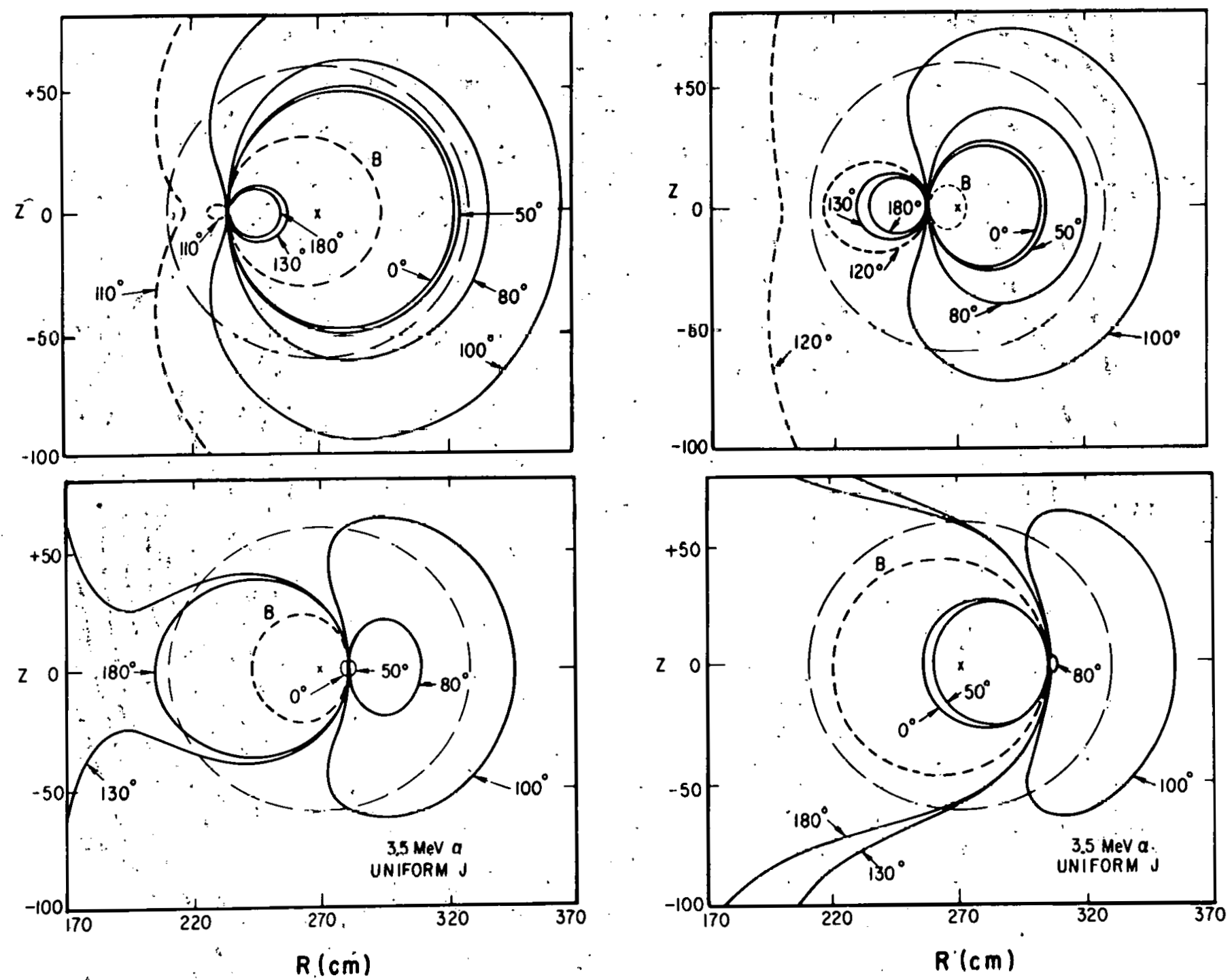

TCT ALPHA ORBITS II=1:0 MA

744601

Fig。2. Alpha Orbit's from Selected Points of Origin. 

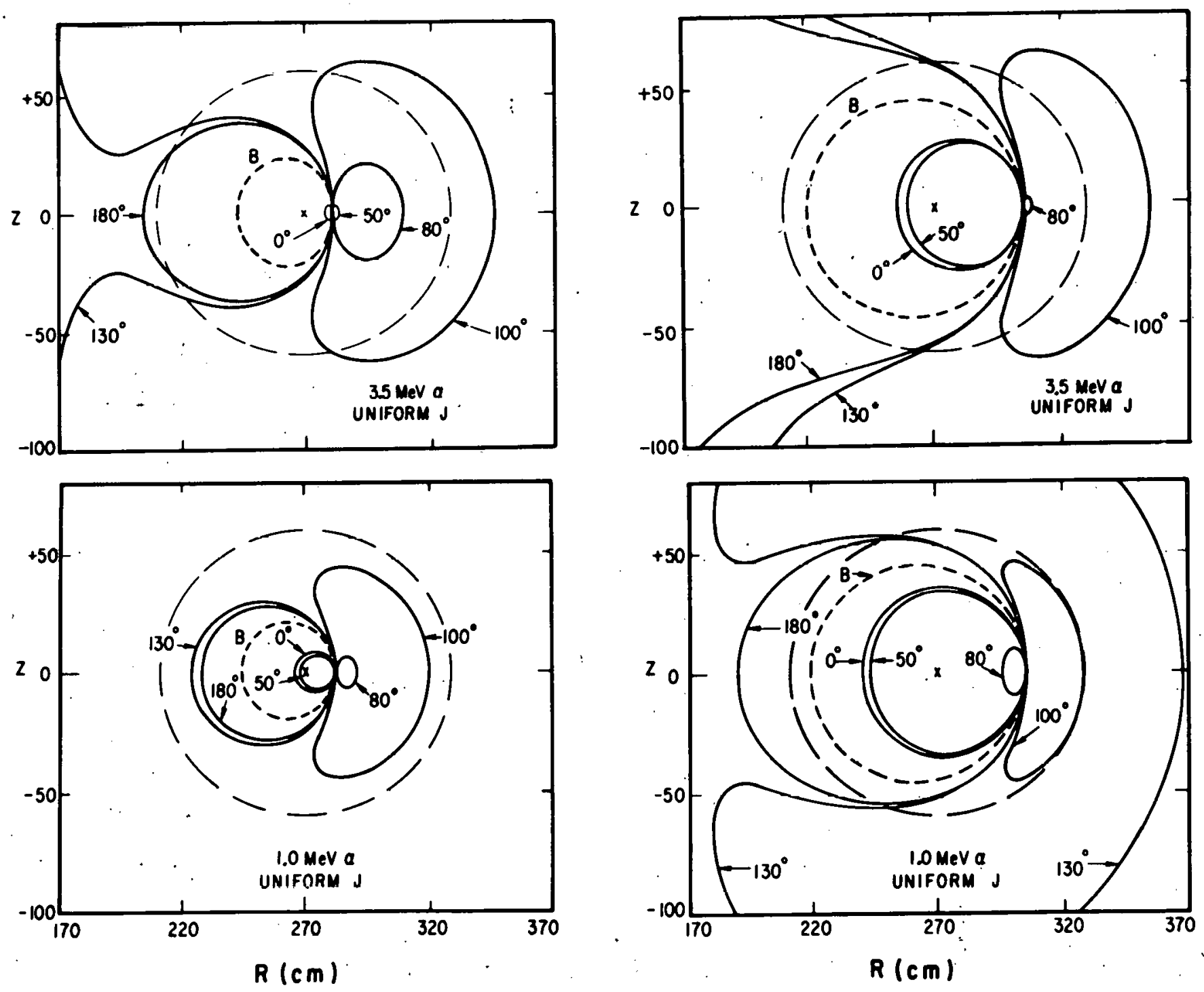

TCT ALPHA ORBITS I $=1.0 \mathrm{MA}$

744600

Fig. 3. Selected Alpha Orbits for Different Energies. 

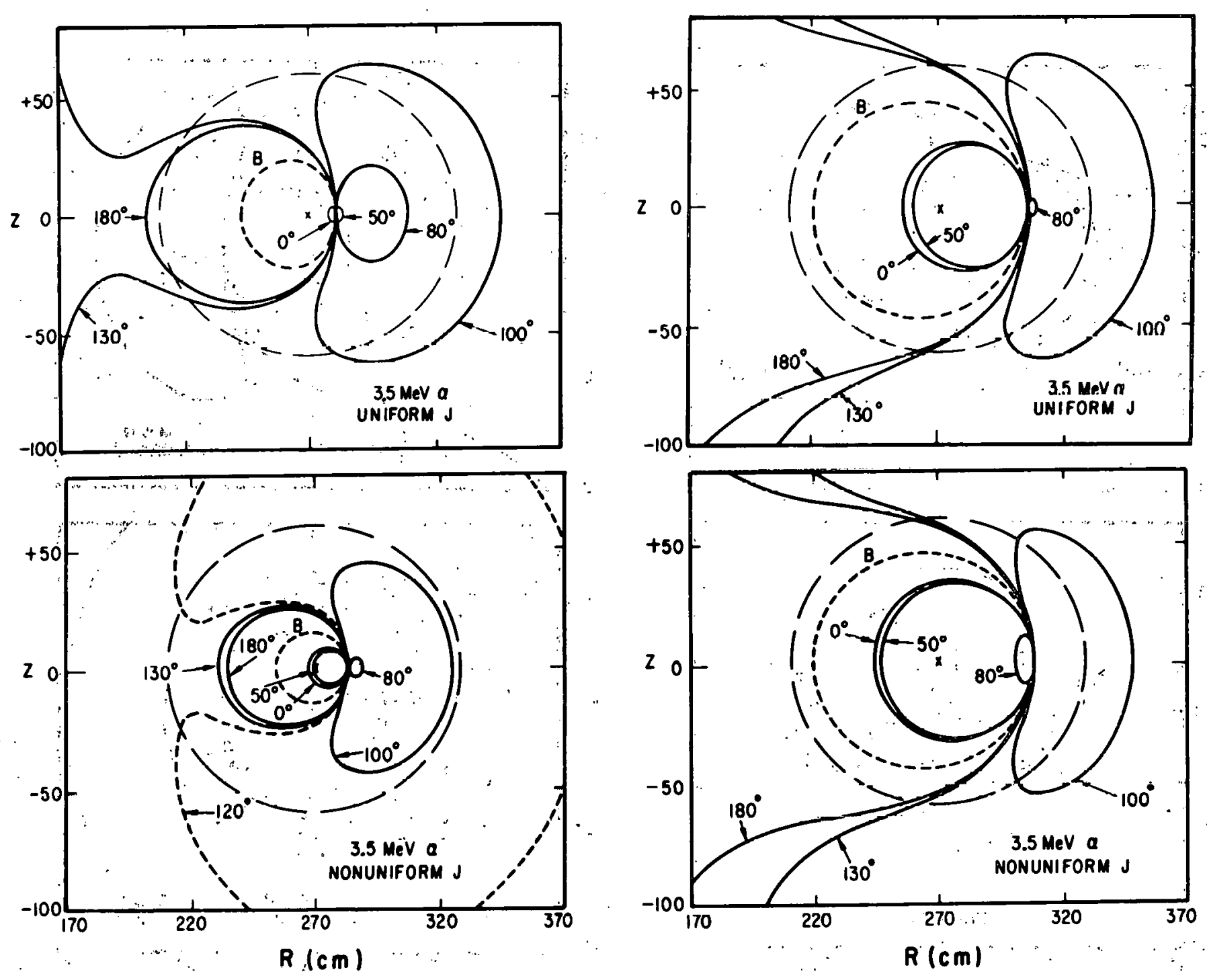

\section{TCT ALPHA ORBITS I $=1.0 \mathrm{MA}$}

744599

Fig. 4. Selected Alpha Orbits for Different Current Distributions. 

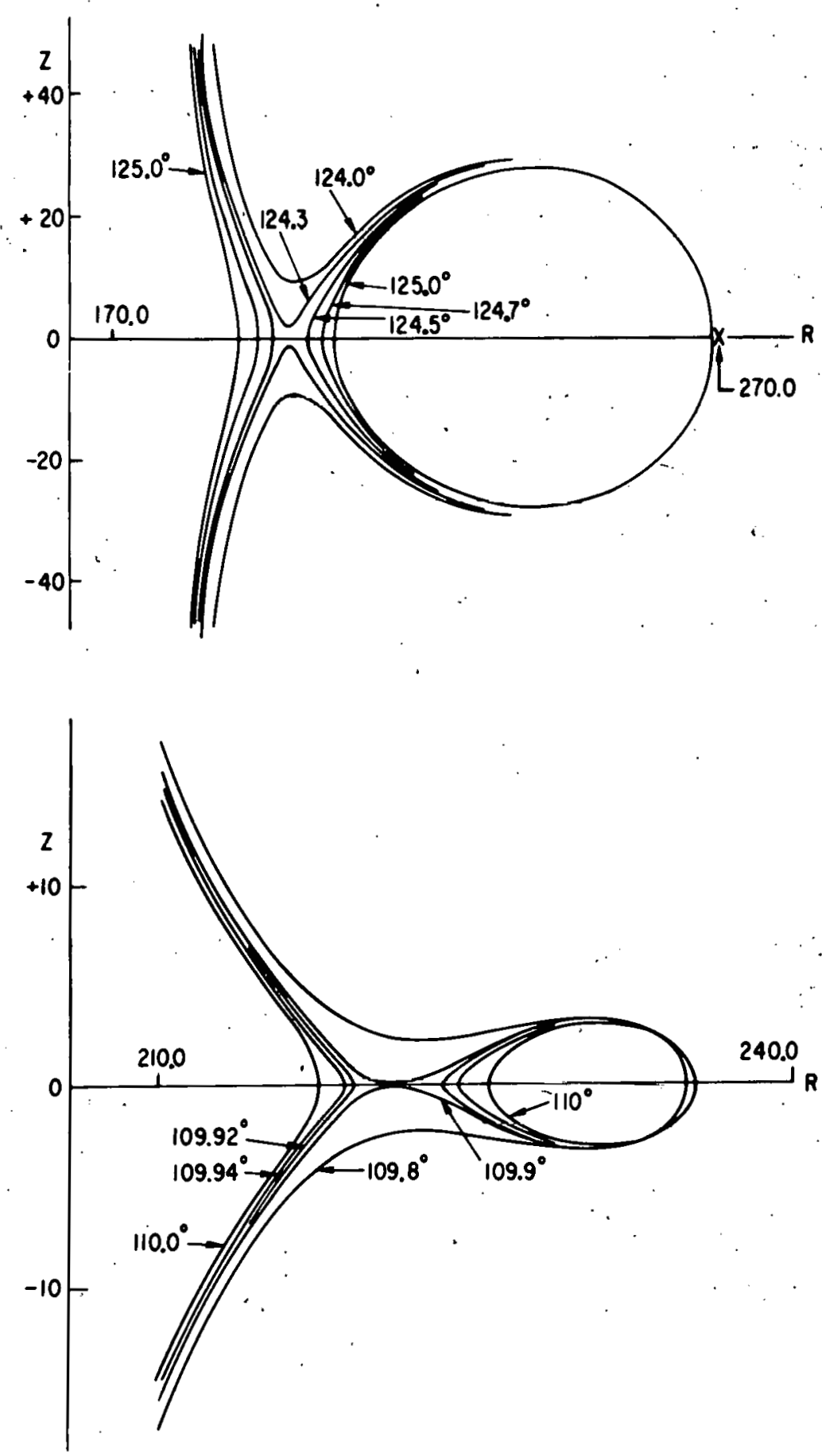

\section{TCT $3.5 \mathrm{MEV}$ ALPHA ORBITS. ALPHA ORBITS NEAR STAGNATION ORBITS} $I=I .0$ MA, UNIFORM J

F1g. 5. Selected Alpha Orbits near Stagnation Orbits. 

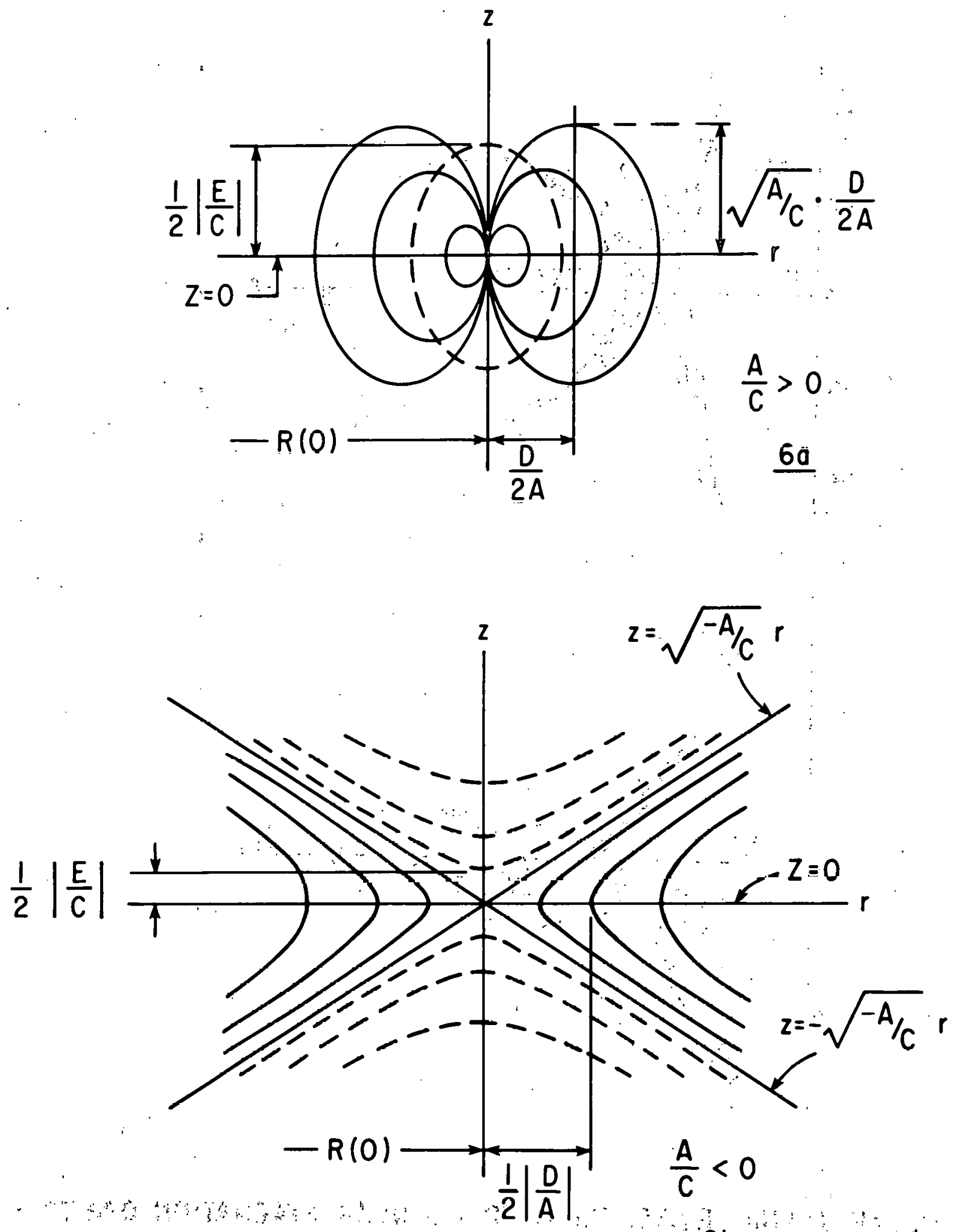

$\underline{6 b}$

753282

Fig. $6:$ Near Stagnation Orbit Patterns. 


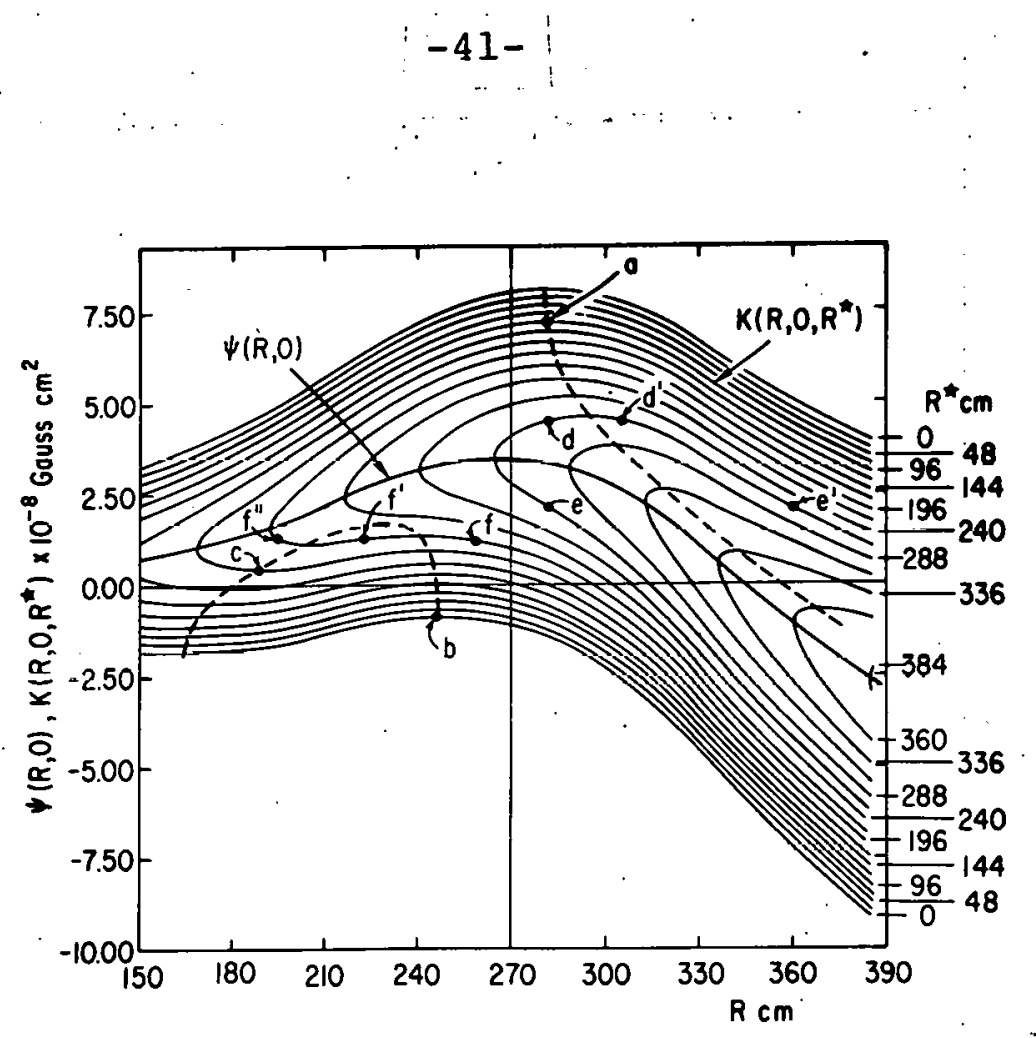

753281

Fig. 7. plot of $K\left(R, 0, R^{*}\right)$ and $\psi(R, O)$ versus $R$.

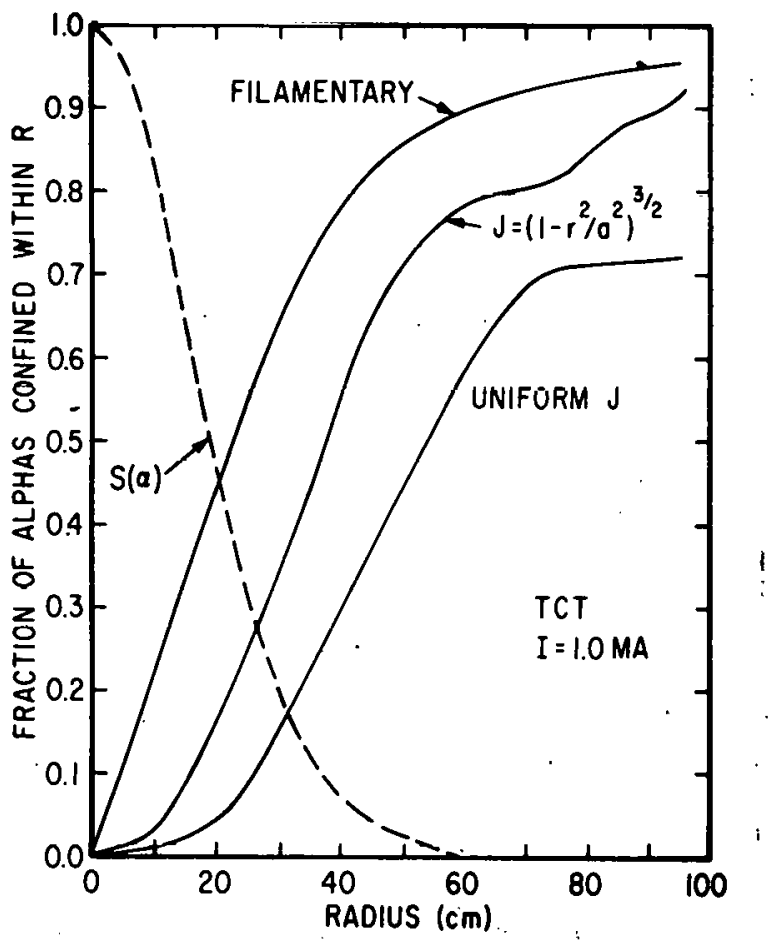

$7532 \overrightarrow{80}$

Fig. 8. Aipha Confinement versus $R$. 


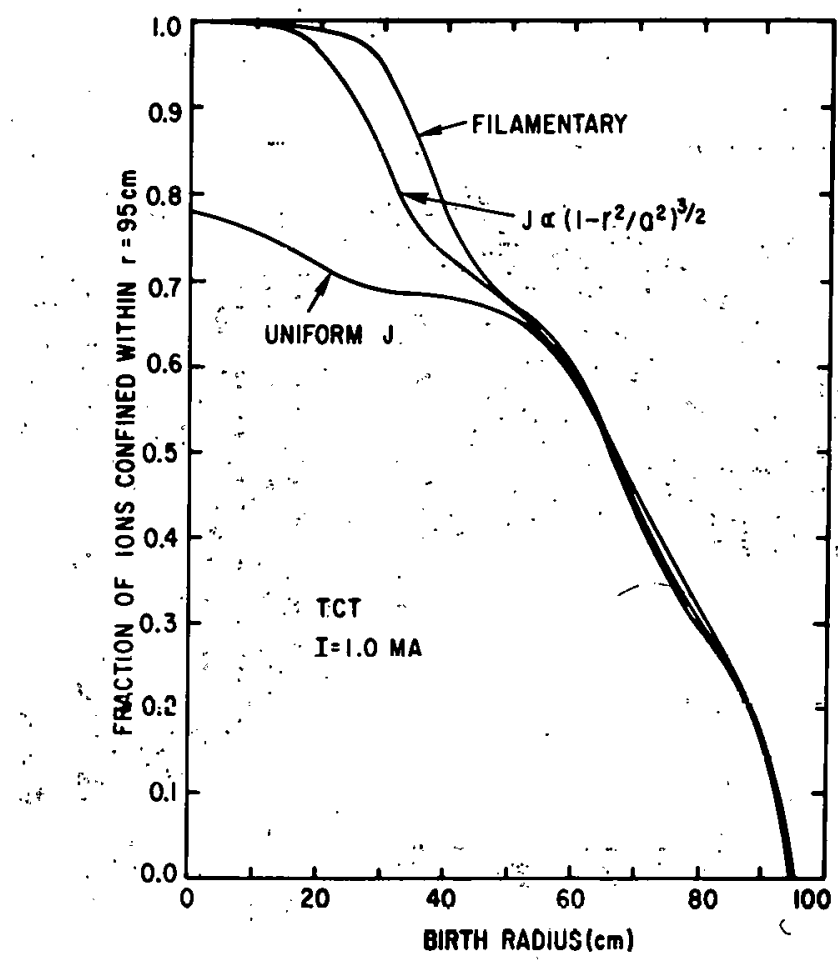

743814

Fig. 9. Alpha Confinement versus Birth Radius $:$

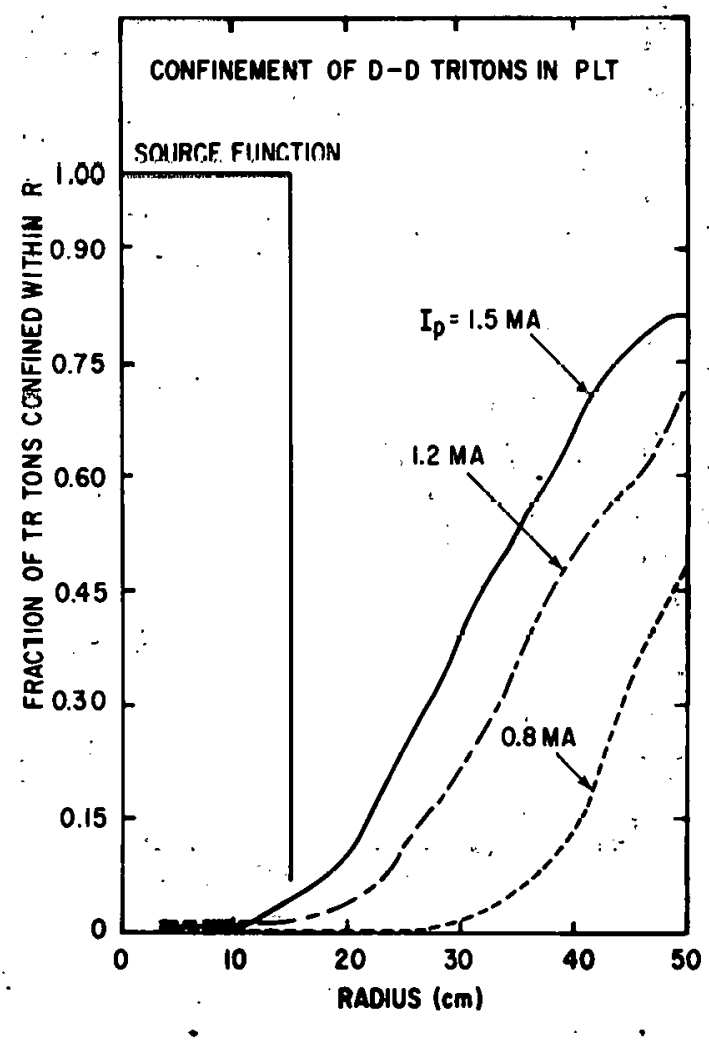

744597

Fig. 10. Trition Confinement versus $R$. 


\section{NOTICE}

This report was prepared as an account of work sponsored by the United States Government. Neither the United States nor the United States Atomic Energy Commission, nor any of their employees, nor any of their contractors, subcontractors, or their employees, makes any warranty, express or implied, or assumes any legal liability or responsibility for the accuracy, completeness or usefulness of any information, apparatus, product or process disclosed, or represents that its use would not infringe privately owned rights. 